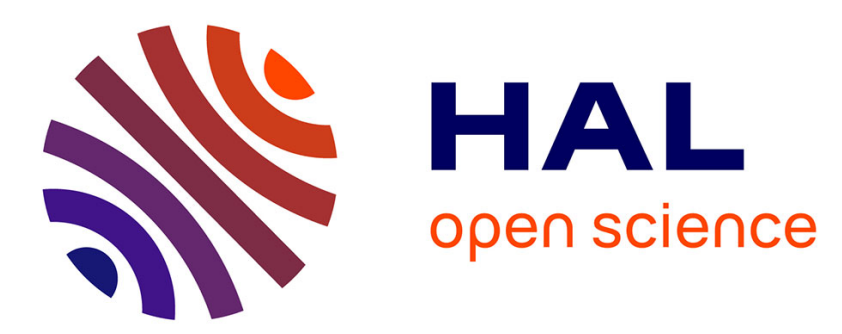

\title{
Grec(que)s contre Égyptien(ne)s dans les enteuxeis ptolémaïques : la question du genre dans les P. Enteux. 79 et P. Enteux. 82 \\ Christine Hue-Arcé
}

\section{To cite this version:}

Christine Hue-Arcé. Grec(que)s contre Égyptien(ne)s dans les enteuxeis ptolémaïques: la question du genre dans les P. Enteux. 79 et P. Enteux. 82. Archimède: archéologie et histoire ancienne, 2018, Archimède $\mathrm{n}^{\circ} 5$. Archéologie et histoire ancienne, 5, pp.165-174. halshs-01826397

\section{HAL Id: halshs-01826397 https://shs.hal.science/halshs-01826397}

Submitted on 29 Jun 2018

HAL is a multi-disciplinary open access archive for the deposit and dissemination of scientific research documents, whether they are published or not. The documents may come from teaching and research institutions in France or abroad, or from public or private research centers.
L'archive ouverte pluridisciplinaire HAL, est destinée au dépôt et à la diffusion de documents scientifiques de niveau recherche, publiés ou non, émanant des établissements d'enseignement et de recherche français ou étrangers, des laboratoires publics ou privés. 


\section{ARCHIMĖDE N'5

1 DOSSIER THÉMATIQUE : HUMOEROTICA

ACTUALITÉ DE LA RECHERCHE : DES FEMMES PUBLIQUES. GENRE, VISIBILITÉ ET SOCIABILITÉ DANS L’ANTIQUITÉ GRECQUE ET ROMAINE

108 Marie AUGIER

Des femmes en capacité d'agir. Introduction au dossier

113 Louise BRUIT ZAIDMAN

«L'enfant du foyer ». Des statues pour les filles et les garçons initiés à Éleusis au nom de la cité

124 Hélène CASTELLI

Pèlerines à Épidaure. Femmes, guérison et publicité dans un sanctuaire panhellénique au IV siècle av. J.-C.

134 Marie-Laure SRONEK

Des femmes invisibles dans l'Athènes classique ? Les effets du travail pour une redéfinition de la place des femmes dans la vie publique

145 Annalisa PARADISO

Femmes lydiennes et crises dynastiques

\section{Sandra PÉRÉ-NOGUÈS}

Sur les traces de Philistis, « reine » de Syracuse : quelques réflexions sur la visibilité des femmes dans les sources monétaires

165 Christine HUE-ARCÉ

Grec(que)s contre Égyptien(ne)s dans les enteuxeis ptolémaïques : la question du genre dans les $P$. Enteux. 79 et $P$. Enteux. 82

175 Claudia BELTRÃO and Patricia HORVAT

The Name of the Vestal, or When a Vestal is Named

185 VARIA

246 LA CHRONIQUE D'ARCHIMÈDE 


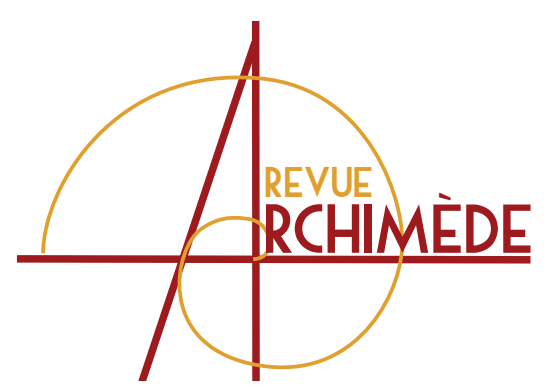

ARCHÉOLOGIE ET HISTOIRE ANCIENNE

\section{GREC(QUE)S CONTRE ÉGYPTIEN(NE)S DANS LES ENTEUXEIS PTOLÉMAÏQUES : LA QUESTION DU GENRE DANS LES P. ENTEUX. 79 ET P. ENTEUX. 82}

Christine HUE-ARCÉ

Docteure en égyptologie de I'Université de Strasbourg christine.huearce@gmail.com

\section{RÉSUMÉ}

Les $P$. Enteux. 79 et 82, deux pétitions grecques du Fayoum adressées au souverain ptolémaïque au III ${ }^{\mathrm{e}}$ s. av. J.-C., ont régulièrement été l'objet de commentaires focalisés sur le genre de leurs protagonistes : dans le premier texte, un Grec porte plainte contre une Égyptienne, tandis que dans le second, c'est une Grecque qui porte plainte contre un Égyptien. Il conviendra d'interroger la pertinence de cette approche genrée par un retour au contenu des textes, puis de mettre en avant les apports de ces pétitions sur le statut des femmes grecques et égyptiennes dans la

\section{MOTS-CLÉS}

Femmes,

genre,

Égypte ptolémaïque,

pétitions,

Fayoum. chôra à la fin du $\operatorname{III}^{\mathrm{e}} \mathrm{s}$. av. J.-C., par l'analyse des actions accomplies par les femmes dans ces deux documents.
P. Enteux. 79 and 82 are two petitions to the Ptolemaic king, written in Greek during the $3^{\text {rd }} \mathrm{c}$. $\mathrm{BCE}$, and originating from the Fayum. Commentaries on these texts have often been focused on the gender of their protagonists: in the first document, a Greek man reports an Egyptian woman, while in the second, a Greek woman reports an Egyptian man. The relevance of this gendered approach will be questioned by returning to the content of the texts. The analysis of women's actions in both petitions will allow the author to highlight their contribution to the understanding of Greek and Egyptian women's status during the $3^{\text {rd }} \mathrm{C}$. BCE in the chôra.

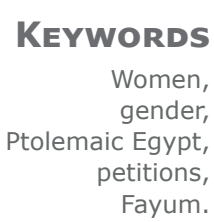


Cet article fait suite à une présentation lors du séminaire Eurykleia tenu à Strasbourg en juin 2017 ; je sais gré à Sandra Boehringer de m'avoir invitée à y prendre part. J'adresse mes remerciements aux « reviewers » anonymes pour leurs corrections et remarques enrichissantes, ainsi qu'à Marie Augier pour son aide et ses précieux conseils. Toute ma reconnaissance à Anne-Emmanuelle Veïsse, pour ses indispensables modifications sur la traduction des textes grecs.

Les enteuxeis ptolémaïques du $\operatorname{III}^{\mathrm{e}} \mathrm{s}$. av. J.-C. [1] sont des pétitions grecques adressées au souverain lagide pour dénoncer des délits variés : vols, spoliations, ou encore agressions, parfois commis ou subis par des femmes [2]. Bien que leur destinataire déclaré soit le roi, ces requêtes étaient en réalité traitées par le stratège et son administration [3], qui pouvaient les rediriger vers l'épistate, afin que ce dernier puisse tenter une conciliation entre les deux parties [4]. En cas d'échec de la conciliation, l'affaire était alors renvoyée devant le stratège, ou aux tribunaux [5]. Ce recours à la justice consultative du souverain nous renseigne non seulement sur le système judiciaire ptolémaïque, mais aussi sur des aspects de la société nilotique à l'époque hellénistique.

Les deux textes qui retiendront notre attention, le $P$. Enteux. 79 et le P. Enteux. 82, exposent des situations analogues : dans les deux cas, le déversement d'un liquide est à l'origine des accusations portées par les auteurs de ces pétitions. Ils présentent par ailleurs un chiasme du point de vue du genre du plaignant et de l'accusé : la première pétition est adressée par un Grec contre une Égyptienne, tandis que la seconde est adressée par une Grecque contre un Égyptien.

Ces textes proviennent tous les deux de I'oasis du Fayoum, la division administrative du nome Arsinoïte à l'époque ptolémaïque, subdivisée en trois mérides : la méris de Thémistos, celle d'Hérakleidès et celle de Polémon. Les papyrus grecs d'Égypte nous ont fourni de nombreux témoignages de Grecs installés dans le Fayoum [6], notamment dans la capitale du nome, Crocodilopolis. Les interactions entre Grecs, et entre Grecs et Égyptiens sont dès lors particulièrement documentées dans cette région d'Égypte, telles les disputes rapportées dans les deux enteuxeis étudiées ici.
Dans le P. Enteux. 79 (11 mai 218 av. J.-C.), le Grec Hérakleidès, porte plainte contre l'Égyptienne Psénobastis : il lui reproche d'avoir versé de l'urine sur lui, alors qu'il passait dans le village.

\section{Recto :}

«Au roi Ptolémée, salut, Hérakleidès, de ceux d'Alexan[drou Nésos], de ceux qui habitent à Crocodilopolis, du nome Arsinoïte. Je suis lésé par Psénobastis, qui habite Psya, du nome susmentionné. Car en l'an 5, d'après le calendrier fiscal, le 21 Phaménoth, je me suis rendu à Psya, du même nome, pour une affaire personnelle. Tandis que je traversais [...] une Égyptienne, nommée, dit-on, Psénobastis, s'est penchée et a versé de l'urine sur mon himation, de sorte que [...] inondé (d'urine) [...]. Alors que je m'étais indigné contre elle, et que je lui faisais des reproches, elle $(\mathrm{m})^{\prime}$ 'a insulté. Quand je l'ai insultée en retour [...], Psénobastis, de sa main droite, ayant tiré le pan

[1] Voir, entre autres, le corpus publié par GUÉRAUD 1931-1932, et les enteuxeis des P. Sorb. III 103-128 (CAdell, Clarysse \& Robic 2011, p. 104-145). Sur la pratique des pétitions dans l'Égypte ptolémaïque, voir GUÉRAUD 1931-1932, p. XIX-LXXXVI, CADELL, Clarysse \& Robic 2011, p. 92-95, Bauschatz 2013, p. $161-217$

[2] Des hypomnêmata, pétitions à destination des agents civils ou militaires du roi (cf. VEÏSSE 2013, p. 82), pouvaient également être adressées par des femmes, comme le montre l'exemple du P. Polit. Jud. 10.

[3] GuÉRAud 1931-1932, p. XXXI-XXXIX ; MÉLÈZEMODRZEJEWSKI 2011, p. 118.

[4] Cf. GuÉRAUD 1931-1932, p. XXXIX.

[5] Cf. GUÉRAUD 1931-1932, p. LXXVI-LXXXI.

[6] Plus d'un cinquième du Fayoum était constitué de terres clérouchiques, exploitées par des Grecs. Cf. THOMPSON 2007, p. 303-310. 
de mon himation qui reposait sur mon épaule, et dont j'étais vêtu, l'a déchiré et frappé, de sorte que ma poitrine s'est retrouvée dénudée let elle m'a craché au visage/ en présence de personnes, que j'ai prises à témoin. Les choses dont je l'accuse, elle les a faites en commettant un acte d'hubris envers moi, et en prenant l'initiative de l'agression. Blâmée par les spectateurs à propos de [sa façon d'agir envers ?] moi, elle m'a laissé ainsi, et elle est rentrée à l'intérieur, d'où elle avait versé I'urine sur moi. Je te prie donc, ô Roi, si bon te semble, [de ne pas me voir avec indifférence] [traité avec] hubris, ainsi sans raison, par une Égy[ptienne], moi qui suis un Grec, et un étranger, mais d'ordonner à Diophanès, le stratège, puisque [...], d'écrire à Sôgénès, l'épistate, qu'il envoie Psénobastis devant [lui (c.-à-d. le stratège), pour être ju]gée contradictoirement avec moi au sujet [de ces faits; et, si les faits] contenus dans ma pétition [sont confirmés], qu'elle reçoive la peine que le stratège déci[dera. Ain]si, grâce à toi, ô Ro[i, j'obtiendrai jus]tice. [Sois heureux] ».

\section{Verso :}

«An 4, 3 Dios, 27 Phaménoth. Hérakleidès contre Psénobastis, à propos d'hubris » [7].

Le $P$. Enteux. 82 (26 février 221 av. J.-C.), est la pétition d'une Grecque contre un Égyptien : Philista, fille de Lysias, accuse Pétékhon, garçon de bains, de I'avoir ébouillantée alors qu'elle était dans la tholos des femmes des bains de Trikomia.

\section{Recto :}

«Au roi Ptolémée, salut, Philista, fille de Lysias, de ceux qui habitent à Trikomia. Je suis lésée par Pétékhon. En effet, je me baignais aux bains dudit village, l'an 1, le 7 Tybi, quand (ce) garçon de bains dans la tholos des femmes, alors que j'étais sortie pour me savonner, ayant apporté les brocs pleins d'eau chaude, (les) a répandus sur moi ; [...] Et il a ébouillanté mon ventre et ma cuisse gauche jusqu'au genou, de sorte qu'il a mis ma vie en danger. L'ayant trouvé, je I'ai livré à Nechthosiris, I'archiphylakitès du village, en présence de Simôn I'épistate. Je te prie donc, ô Roi, si bon te semble, ayant trouvé refuge comme suppliante auprès de toi, de ne pas me voir avec indifférence traitée ainsi en dépit des lois, moi qui vis de mes mains, mais d'ordonner à Diophanès, le stratège, d'écrire à Simôn V'épistate/, et à Nechthosiris, le policier, qu'ils amènent devant lui Pétékhon [a]fin que Diophanès examine l'affaire, pour que, en cherchant refuge auprès de toi, ô Roi, le commun bien[fai]teur de tous, j'obtienne justice. Sois heureux ».

« À Simôn : envoie l'accusé. An 1, 28 G[orpi]aios, 12 Tybi ».
Verso :

«An 1, 28 Gorpiaios, 12 Tybi. Philista contre Pétékhon, garçon de bains, à propos de brûlures » [8].

Les $P$. Enteux. 79 et 82 ont régulièrement fait I'objet de commentaires focalisés sur le genre du pétitionnaire et de l'auteur des faits reprochés. La pertinence de cette approche doit être interrogée par un retour au contenu des textes : quelles informations sont réellement mises en avant par leurs auteurs ? Il conviendra également de souligner les apports des deux pétitions sur le statut des femmes grecques et égyptiennes dans la société de la chôra à la fin du III $^{\mathrm{e}}$ s. av. J.-C.

\section{UNE HISTORIOGRAPHIE FOCALISÉE SUR L'OPPOSITION HOMME/FEMME}

Les $P$. Enteux. 79 et 82 ont fait I'objet d'une editio princeps par ]. Lesquier en 1912 [9]. Réédités dans le corpus des enteuxeis du $\mathrm{III}^{\mathrm{e}} \mathrm{s}$. av. J.-C. par O. Guéraud en 1931, ils ont depuis lors été régulièrement commentés, notamment dans des articles consacrés aux violences par et contre des femmes, à la lumière d'une opposition entre le genre de l'accusateur/trice et celui de l'accusé(e).

Dans ces publications, le genre des protagonistes rentre toujours en compte; bien que d'autres aspects soient parfois discutés, l'insistance des commentateurs porte essentiellement sur la question du genre, tant dans la description des textes et leur analyse, que dans les conclusions générales qui en sont tirées [10]. Le $P$. Enteux. 79 a notamment fait l'objet d'un tel commentaire par M. Parca en 2002 [11], dans un article consacré aux violences exercées par et contre les femmes dans les papyrus documentaires d'Égypte gréco-romaine. La chercheuse analyse divers facteurs dans le déversement d'urine par Psénobastis

[7] Traduction GuÉRAUD 1931-1932, p. 191-192, légèrement modifiée.

[8] Traduction GuÉRAUD 1931-1932, p. 199-200, légèrement modifiée.

[9] LeSQUIER 1912. P. Lille II 24 (= P. Enteux. 79) et $P$. Lille II 33 (= P. Enteux. 82).

[10] Je me concentrerai ici sur une présentation des commentaires axés sur le genre. Pour les analyses des deux papyrus dans d'autres optiques, cf. notamment : pour le P. Enteux. 79, LeWIS 1986, p. 59, BAUSCHATZ 2013, p. 161-163 ; pour le P. Enteux. 82, LEWIS 1986, p. 59.

[11] PARCA 2002, p. 284-288. 
sur Hérakleidès - qu'elle considère comme volontaire [12] - et dans la dispute qui s'ensuit, tels que I'ethnicité et la barrière de la langue. Le genre de Psénobastis et d'Hérakleidès tient néanmoins pour elle une place importante : $M$. Parca évoque en effet une double profanation d'un Grec humilié par une Égyptienne, et d'un homme humilié par une femme [13].

De même, dès sa première édition, le $P$. Enteux. 82 a fait l'objet d'une traduction par J. Lesquier, plus tard reprise par $O$. Guéraud, qui trahissait son interprétation du texte. En effet, dans le texte grec, Pétékhon est simplement désigné par son activité au sein des

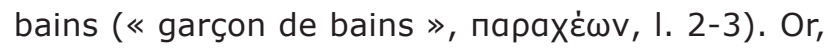
J. Lesquier, dans le corpus des $P$. Lille II, traduisait le passage ainsi : «quand un homme, garçon de bains dans l'étuve des femmes » [14].

Pour B. Legras, le déversement d'eau bouillante par Pétékhon sur Philista - qu'il range dans la catégorie des «violences des hommes contre des femmes »constitue une attaque volontaire de l'homme égyptien sur la femme grecque [15]. S'il évoque la possibilité d'une simple confusion de brocs par Pétékhon, le chercheur ne s'attarde pas sur cette hypothèse et semble retenir l'idée d'un geste volontaire, dont il questionne les motivations : «Est-il incompétent ou distrait ? On pourrait dans ce cas imaginer que Pétéchôn a pu être troublé par la vue de cette (belle ?) femme aux bains... Pétéchôn en voulait-il spécialement à Philista ? Leur contentieux est-il alors personnel ? Est-ce l'expression d'un conflit masculin/ féminin ? Faut-il y voir plutôt l'expression d'un heurt entre un Égyptien et une Grecque ? Ne serait-il que le bras malveillant d'une personne en voulant à la baigneuse ? » [16]. L'action de Pétékhon pourrait ainsi avoir été le geste volontaire d'un homme sur une femme, en raison de cette différence de genre. $B$. Legras suggère également une confusion des brocs d'eau (alors involontaire), qui aurait pu être provoquée par la vue du corps féminin de Philista.

[12] PARCA 2002, p. 285.

[13] « To be befouled by another person's urine was to be defiled, and to our plaintiff the physical pollution was but the visible manifestation of a deeper desecration: that of a Greek sullied by an Egyptian, and that of a man tainted by a woman » (PARCA 2002, p. 285-286).

[14] LESQUIER 1912, p. 180 ; traduction reprise par GuÉRAUD 1931-1932, p. 199.

[15] LEGRAS 1999, p. 229, évoque en effet des « brûlures infligées volontairement à une femme dans un bain public ».

[16] LEGRAS 1999, p. 230.
Je reviendrai plus tard sur la question d'un « conflit masculin/féminin ». Je souhaite néanmoins m'arrêter quelques instants sur l'hypothèse d'un «trouble » ressenti par Pétékhon [17] : le garçon étant affecté dans la tholos des femmes, le corps de Philista n'était sans doute pas le premier porté à sa vue. Si de telles erreurs survenaient à chaque corps féminin aperçu - eût-il été particulièrement beau, difforme, jeune ou marqué par le poids des années - bien d'autres incidents similaires auraient été relevés dans les bains de Trikomia, et le jeune homme n'aurait guère longtemps conservé son emploi.

\section{UNE IMPORTANCE RÉELLE DE L'IDEN- TITÉ DE SEXE SELON LES PLAIGNANTS?}

Les interprétations des $P$. Enteux. 79 et 82 centrées sur les rapports entre le pétitionnaire et l'auteur des faits reprochés s'appuient sur la différence de sexe entre les protagonistes de chaque texte. Néanmoins, une telle analyse, isolée, est-elle toujours pertinente? L'approche avec l'outil du genre s'avère fructueuse ; en effet, quand les identités sont historicisées, la définition de la démarche peut être résumée en ces termes : «La "différence des sexes" relève d'une interprétation sociale et culturelle des différences perçues entre le corps des hommes et celui des femmes. Pour l'historien de la Grèce et de la Rome antiques, le genre est un outil d'analyse permettant de mettre au jour les catégories propres aux Anciens dans leur perception et leur définition d'un corps ou d'un comportement, sans présupposer une priorité d'un critère sur un autre » [18].

Dans ce contexte, la focalisation sur l'identité de sexe est utile mais demande une contextualisation : cette différenciation passait-elle, pour les Anciens, avant les autres différenciations ? Pour répondre à cette interrogation, il convient de se détacher des commentaires antérieurs et de retourner au contenu

[17] Hypothèse reprise par B. Legras d'une suggestion moins précise de RowLANDSON 1998, p. 172, qui s'étonne en effet qu'un homme/garçon soit affecté à la tholos des femmes alors que les Grecs étaient très protecteurs de la pudeur des femmes; J. Rowlandson rappelle néanmoins que pour les Grecs, les serviteurs et esclaves « did not count as people for purposes of modesty, however much this might trouble the servants 》 (I'italique est ajouté par l'auteure du présent article).

[18] Sandra Boehringer et Violaine Sebillotte Cuchet, lors de la journée « L'histoire du corps dans I'Antiquité : bilan historiographique » organisée par la SoPHAU le 25 mai 2013, publiée dans les Dialogues d'histoire ancienne (Boehringer \& Sebillotte Cuchet 2015). 
des pétitions, afin de relever les informations mises en avant par leurs auteurs.

\section{P. Enteux. 79}

Les informations qui ressortent du $P$. Enteux. 79 concernent avant tout I'origine géographique et ethnique d'Hérakleidès et de Psénobastis. L'insistance d'Hérakleidès dans sa pétition porte en effet sur son statut de Grec. Comme le veut I'usage des pétitions [19], I'homme indique son lieu de domicile, mais aussi son village d'origine, Alexandrou Nésos [20] (I. 1), en partie peuplé de clérouques [21]. Hérakleidès est par ailleurs vêtu d'un himation $(1.4,6)$ : ce vêtement drapé, typique du monde grec ancien, marque visuellement son appartenance à la communauté hellène. Enfin, I'homme s'identifie lui-même comme un Grec, soulignant dans sa pétition au souverain : « Moi

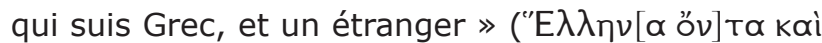
छ́vंov, l. 9-10).

Les informations fournies par Hérakleidès sur Psénobastis sont plus succinctes : la femme habite à Psya (I. 2), le lieu de l'incident, village situé dans la méris d'Hérakleidès, au nord-est du Fayoum. Le Grec n'indique pas le patronyme de l'Égyptienne, et l'anthroponyme Psénobastis est vraisemblablement une mauvaise retranscription : Psénobastis correspond en effet au démotique $P_{3}-\check{s} r-n$-B $B_{3}$ st.t, un nom masculin, là où pour une femme le féminin $T_{3}-\check{s} r . t-n-B 3 s t . t$ (Sénobastis) est attendu [22]. La seule information claire qu'Hérakleidès nous fournit sur Psénobastis est son ethnicité : il s'agit d'une Égyptienne (Aijumtía, I. 4, 9).

À travers les informations apportées par Hérakleidès dans sa pétition, il ressort une opposition en termes d'appartenance ethnique entre Psénobastis et Hérakleidès, soulignée par ce dernier : I'une est Égyptienne, tandis que l'autre est Grec [23]. Hérakleidès mentionne également qu'il est un étranger ( $\xi$ Évov, l. 10). En effet, il n'habite pas le même village que Psénobastis, mais pis encore, ils vivent dans deux mérides distinctes du Fayoum [24]. Or, les solidarités villageoises sont importantes dans l'Égypte hellénistique : comme I'a montré A. Helmis, le refus de l'autre s'applique aux différences locales, et il est fréquent que des conflits impliquent des villages différents [25] ; « le village [...] est aussi une géographie sociale » [26].

Par ailleurs, une opposition de statut social est également notable entre Psénobastis et Hérakleidès : I'homme grec est vêtu d'un himation, un vêtement drapé. Or, porter un tel vêtement ne permet pas d'effectuer un travail manuel, ce qui invite à supposer qu'Hérakleidès est issu d'un milieu social aisé. Au contraire, au moment de l'incident, Psénobastis vide de l'urine : qu'il s'agisse de sa propre urine, ou de celle d'un maître si elle était une servante, cette action dénote un niveau social inférieur.

Dans sa pétition, Hérakleidès accuse Psénobastis

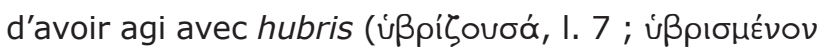

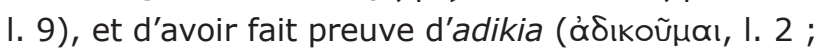

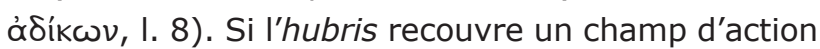
large, généralement rendu par la notion moderne de « violence » [27], elle constitue avant tout un crime dans l'Égypte lagide [28] : Hérakleidès n'accuse pas seulement Psénobastis d'avoir agi avec démesure, il lui reproche un délit juridiquement reconnu et sanctionné par le droit ptolémaïque, rencontré dans plusieurs autres plaintes [29]. De même, le Grec accuse l'Égyptienne d'avoir agi avec adikia : dans la documentation papyrologique légale, dire d'une action qu'elle est « injuste », consiste en réalité à contester sa légalité [30]. Hérakleidès appuie ainsi sa plainte
[19] Cf. VeÏsSE 2013, p. 83.

[20] Sur la mention du lieu de domicile dans les pétitions grecques, cf. VEÏSSE 2012, p. 48.

[21] Cf. Uebel 1968, no. 357-361.

[22] PARCA 2002, p. 287 suggère qu'Hérakleidès a appris le nom de Psénobastis par une tierce personne, et y voit un indice que le Grec et l'Égyptienne se sont peutêtre disputés en parlant chacun sa propre langue, ou en ayant une connaissance très limitée de la langue de I'autre. Voir également VEÏSSE 2012, p. 50.

[23] VEÏSSE 2012, p. 49-50, souligne I'exceptionnalité de cette «stratégie identitaire » dans les textes ptolémaïques.

[24] Le cas d'Hérakleidès n'est pas sans évoquer celui de Dionysios dans le $P$. Hels. I 2, roué de coups, puis expulsé du bain où il se baignait, jusqu'aux portes du village : REDON 2015, p. 79-80 suggère que l'agression de Dionysios a été amplifiée par le fait qu'il était un « homme de passage ». Ce cas de figure d'un agresseur local et d'une victime de passage apparaît également dans la plainte du P. Enteux. 83 (REDON 2015, p. 80-81).

[25] HeLMIS 1986, p. 63.

[26] HeLmis 1986, p. 67.

[27] La notion d'hubris recouvre un spectre large de significations, et désigne chez Aristote les « motivations profondes d'un acte criminel » (HELMIS 1986, p. 21). Dans la documentation ptolémaïque, ce terme « connote, en règles générales, des attaques personnelles mais il ne nous est pas possible d'en affirmer davantage la signification » (HELMIS 1986, p. 22).

[28] TAUBENSCHLAG 1955, p. 435-442.

[29] La notion d'hubris est notamment employée dans les enteuxeis des $P$. Sorb. III 112, P. Enteux. 74, 75 et 78 , ou encore dans le $P$. Gurob 2 ; noter que dans ce dernier texte, c'est également une femme qui est accusée d'hubris.

[30] Cf. MÉLÈZE-ModRZEJEWSKI 2011, p. 94. 
sur des notions juridiques précises, et non sur le seul sentiment d'humiliation ou d'outrage.

Il ressort ainsi du $P$. Enteux. 79 l'importance d'une double opposition entre les deux protagonistes, ethnique et sociale, avec une accentuation de la différence d'ethnie, et des accusations fondées sur des aspects spécifiques du droit ptolémaïque. Le genre des protagonistes est évidemment indiqué par l'utilisation grammaticale du féminin et du masculin dans ce texte ; aucune insistance n'est pourtant portée par Hérakleidès sur cette différence de genre entre Psénobastis et lui-même [31].

Un facteur important de la plainte d'Hérakleidès pourrait être la demande d'une sanction pécuniaire à la fin du texte : le but de sa pétition contre Psénobastis est en réalité de recevoir un dédommagement financier.

Une phrase du $P$. Enteux. 79 est en ce sens particulièrement révélatrice : Hérakleidès insiste sur le fait que c'est Psénobastis qui est à l'origine de la dispute. En effet, après le déversement de l'urine sur Hérakleidès, Psénobastis et le Grec échangent des insultes, et la femme, écrit Hérakleidès, « ayant tiré le pan de mon himation qui reposait sur mon épaule, et dont j'étais vêtu, l'a déchiré et frappé, de sorte que ma poitrine s'est retrouvée dénudée » (I. 6-7). Elle a commis le délit d'hubris, souligne-t-il, « en prenant I'initiative

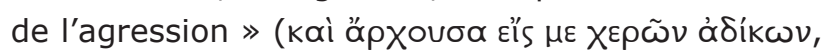
I. 7-8). Hérakleidès reproche ainsi à Psénobastis d'être responsable de l'escalade de la dispute. Cette accusation ne joue pas un moindre rôle dans la pétition du Grec : si Hérakleidès parvient à faire porter la responsabilité de ce différend à Psénobastis, l'estimation par le stratège du dédommagement qu'il recevra sera certainement plus importante [32]. Il convient néanmoins de souligner qu'à aucun moment Hérakleidès n'accuse explicitement Psénobastis d'avoir volontairement déversé I'urine sur lui. L'accusation d'hubris concerne l'envenimement de la dispute, et non le geste qui en est à l'origine.

Le moteur premier de la dénonciation d'Hérakleidès pourrait ainsi avoir été la volonté d'un dédommagement financier [33], plus que la volonté d'une rétribution face au sentiment de « profanation » qu'évoquait $M$. Parca.

\section{P. Enteux. 82}

Dans sa pétition contre Pétékhon pour l'avoir ébouillantée aux bains [34] de Trikomia, Philista avance plusieurs faits pour appuyer sa plainte. Elle insiste notamment sur le danger qui a pesé sur sa vie : «il a ébouillanté mon ventre et ma cuisse gauche jusqu'au genou, de

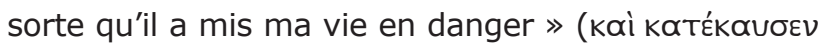

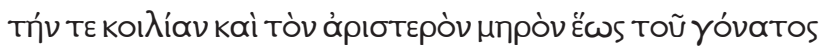

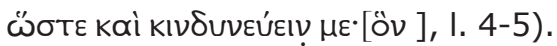

Cette insistance va de pair avec les conséquences financières du geste de Pétékhon, qui sont sous-entendues par Philista. La femme indique en effet qu'elle

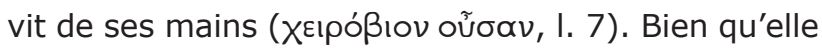
n'établisse pas de lien direct entre son travail manuel et les blessures subies, cette information sur son occupation suggère des retombées financières : si sa vie a été mise en danger par les brûlures infligées, Philista n'a pas pu travailler depuis l'incident des bains, et n'a donc pas eu la possibilité de gagner sa vie. Philista ne demande pas clairement de dédommagement au souverain, mais parle «d'obtenir justice » (I. 10). En réalité, les textes d’Égypte hellénistique - tant démotiques que grecs - relatifs à des actes de violence ne réclament pas de châtiments corporels : lorsque la sanction demandée est explicitée, il s'agit de peines pécuniaires [35]. Il est ainsi vraisemblable que Philista ait espéré un dédommagement matériel, insistant pour ce fait sur la fragilité de sa situation financière [36]. L'auteure du $P$. Enteux. 82 ne souligne pas la différence d'ethnie avec son agresseur. L'origine ethnique
[31] L'absence d'insistance sur la différence sexuelle me semble bien perceptible dans la plainte d'Hérakleidès. En effet, la différence d'origine ethnique et géographique est déjà clairement identifiable par la mention de l'ethnique et du village d'où est originaire Hérakleidès. Pourtant, le Grec insiste tout de même sur son statut d'étranger en se disant xénos. S'il avait souhaité, de la même manière, insister sur le genre féminin de son agresseur, ne l'aurait-il pas désignée comme une femme, ne se limitant pas à l'emploi de la forme féminine du terme Aiguptia?

[32] Les prix de certains vêtements, tels le chiton et le vêtement in-nw, pouvaient atteindre entre deux et dix fois le salaire moyen de I'ouvrier et du paysan à l'époque ptolémaïque. Cf. CLARYSSE \& LANCIERS 1989, p. 118,120 .

[33] Dans les textes égyptiens et grecs d'époque gréco-romaine, les plaintes à la suite d'actes de violence sont fréquemment liées à des considérations économiques, qu'il s'agisse d'un dédommagement financier ou d'un bien à restituer. Ces aspects économiques constituent en réalité le premier facteur de dénonciation auprès des institutions juridiques - ou en tout cas de la mise par écrit des disputes portées devant ces instances. Cf. HUE-ARCÉ 2017, p. 145-146 ; LEGRAS 1999.

[34] Sur les violences aux bains dans l'Égypte ptolémaïque, voir REDON 2015, p. 78-81.

[35] Il s'agit d'une conclusion ressortie de l'étude des textes d'époque ptolémaïque et romaine, menée dans le cadre de ma thèse de doctorat sur la violence interpersonnelle dans la documentation égyptienne du Nouvel Empire et d'époque gréco-romaine, soutenue à Strasbourg en 2015, et encore inédite à ce jour : HUEARCÉ 2015, p. 274-275, 281-285.

[36] Cf. FAUCHER \& REDON 2014, p. 849, n. 64 ; REDON 2015, p. 68-69, n. 47. 
de Philista et Pétékhon est identifiable non par des informations fournies par Philista dans sa pétition, mais par l'onomastique des deux individus : Pétékhon est un anthroponyme typiquement égyptien, tandis que Philista est un nom grec, de même que l'est son patronyme, Lysias. Si les Égyptiens portaient parfois des noms grecs, au $\operatorname{III}^{\mathrm{e}} \mathrm{s}$. av. J.-C. cette pratique est encore peu répandue, et I'onomastique demeure un bon indicateur de I'appartenance ethnique [37]. Pourtant, contrairement à Hérakleidès dans le $P$. Enteux. 79, Philista ne fait aucune mention de ces ethnies distinctes.

De même que la plainte d'Hérakleidès, la pétition de Philista ne met en avant ni le genre de la plaignante, ni celui de son agresseur pour dénoncer les faits (contrairement à ce que laissait supposer la traduction de J. Lesquier [38]). L'unique circonstance aggravante soulignée est l'impact sur le travail quotidien de la femme grecque et le danger qui a pesé sur sa vie, tandis que la seule information apportée sur Pétékhon est sa fonction de «garçon de bains » [39].

Pour B. Legras [40], cet incident constitue vraisemblablement une attaque volontaire d'un homme égyptien sur une femme grecque, bien qu'il évoque la possibilité d'une confusion des brocs d'eau par Pétékhon. Néanmoins, rien ne suggère dans cette plainte que Philista reproche au garçon de bains d'avoir volontairement versé de l'eau bouillante. Philista était sortie de l'eau pour se savonner : il est possible que Pétékhon ait versé de l'eau sur elle pour rincer le savon, à sa demande, sans s'être rendu compte que l'eau était trop chaude [41]. Au contraire du $P$. Enteux. 79, le $P$. Enteux. 82 ne fait pas état d'hubris, d'insultes ou de comportement violent.

En réalité, Philista ne questionne à aucun moment les motivations de Pétékhon, ni l'éventualité d'une préméditation ou d'un geste volontaire. L'insistance est portée sur les conséquences des brûlures et sur les répercussions financières sous-jacentes.

\section{FEMMES GRECQUES ET ÉGYPTIENNES DANS LA CHÔRA AU III' S. AV. J.-C.}

Ainsi, l'identité de sexe des protagonistes de ces pétitions ne rentre pas directement en compte pour les plaignants. Le statut social, I'origine ethnique et géographique, les conséquences économiques du geste ou encore la mise en danger de la vie sont les facteurs que les auteurs des deux plaintes mettent en avant. Il est indéniable que ces documents constituent la plainte d'un homme contre une femme, et la plainte d'une femme contre un homme ; néanmoins, le critère du genre n'est aucunement souligné comme facteur aggravant. La différence sexuelle constitue dans ces textes une simple « différence parmi d'autres » [42], et révèle ainsi un régime de genre propre à cette société. Ces plaintes ne sont pourtant pas dénuées d'informations sur I'histoire du genre, et nous instruisent sur les actions de femmes dans la chôra égyptienne du III $^{\mathrm{e}}$ s. av. J.-C.

Si les informations apportées par le P. Enteux. 79 sur le statut des femmes dans le Fayoum à l'époque de sa rédaction sont peu abondantes, elles ne sont pas pour autant inexistantes. Le statut social inférieur de Psénobastis peut ainsi être déduit de son activité : elle vidait de l'urine depuis l'intérieur d'une maison, ce qui peut indiquer qu'elle était une servante, ou ne possédait pas de serviteurs pour effectuer cette tâche. En dépit de cette indication sur le statut social de Psénobastis, et de son genre, Hérakleidès porte plainte directement contre elle, semblant ainsi la considérer comme disposant de ses pleines capacités juridiques.

Le $P$. Enteux. 82 se révèle quant à lui particulièrement riche en renseignements : dans cette pétition, Philista, une femme grecque, se plaint directement au souverain, sans être représentée par un homme. De la même manière, elle mentionne dans sa requête avoir dans un premier temps dénoncé Pétékhon auprès de I'archiphylakitès, le chef de la police du nome. Cette enteuxis nous montre ainsi une Grecque d'Égypte effectuer une démarche judiciaire elle-même, en son nom propre, sans être représentée par un kurios - époux, père, frère ou fils. Cette autonomie de Philista doit-elle être expliquée par un veuvage ou I'absence de parent masculin ? Rien dans le texte du $P$. Enteux. 82 ne suggère que I'action autonome de Philista soit due à une situation familiale particulière. Aucune justification ou explication n'est jugée nécessaire par l'auteure de la plainte : il ne s'agit pas

[37] Peremans 1970, p. 213-223.

[38] Cf. supra.

[39] Sur les « garçons de bains », dont une des fonctions était de verser l'eau sur les clients des bains, cf. FAUCHER \& REDON 2014, p. 842-843.

[40] LEGRAS 1999, p. 229.

[41] L'hypothèse d'une « simple maladresse » de Pétékhon est également retenue par REDON 2015, p. 79.

[42] Cf. Boehringer \& Sebillotte Cuchet 2011, p. 22. Voir à ce propos la notion d'intersectionnalité, qui insiste sur l'importance d'une prise en compte de l'ensemble des facteurs déterminants - origine, statut social, genre, etc. ; sur l'utilisation de ce concept en égyptologie, cf. MATIć 2016, p. 1-22. 
d'une situation exceptionnelle, qui nécessiterait d'être motivée. La pétition du $P$. Enteux. 82 nous indique ainsi qu'une femme grecque d'Égypte, d'un niveau social loin d'être supérieur [43], pouvait effectuer des démarches juridiques en toute autonomie, sans avoir à s'en justifier auprès des autorités compétentes.

En réalité, la démarche de Philista est loin d'être inhabituelle : les femmes de la chôra, grecques et égyptiennes, bénéficiaient d'une certaine autonomie pour mener leurs affaires, établir des contrats, ou déposer des plaintes. Les pétitions adressées par des femmes pour dénoncer des violences ou des préjudices économiques sont plus fréquentes qu'on ne pourrait le croire : E. Scheerlinck a ainsi relevé 71 pétitions adressées par des femmes sur toute l'époque hellénistique [44]. La chercheuse belge a souligné l'autonomie des femmes dans ces plaintes, ainsi que leur faculté à gérer des affaires économiques et à engager des poursuites contre d'autres femmes ou des hommes sans intervention explicite de membres de leur parenté [45]. L'ingérence du kurios était nécessaire pour certains actes établis par les femmes grecques d'Égypte ; néanmoins, les enteuxeis pouvaient être adressées en toute indépendance, sans assistance du tuteur [46].

\section{CONCLUSION}

Si les pétitions des $P$. Enteux. 79 et 82 ont fait I'objet de commentaires principalement focalisés sur le genre de leurs protagonistes, le retour au contenu des textes permet de montrer que cette interprétation est en réalité erronée : le genre du défendeur et du plaignant $n^{\prime} y$ est pas mis en avant, il ne s'agit pas d'un facteur considéré comme aggravant les incidents survenus. Bien que le genre des protagonistes soit grammaticalement identifiable, les mots « homme » et « femme » ne sont ainsi pas employés par les auteurs des deux plaintes.

[43] Cf. le passage du $P$. Enteux. 82 où Philista indique qu'elle «vi[t] de [ses] mains », I. 7.

44] SCHEERLINCK 2011-2012, p. 166. Voir également VEÏSSE 2013, p. 85, qui notait que $15 \%$ des 330 pétitionnaires qu'elle a identifiés dans les enteuxeis et hypomnêmata ptolémaïques sont des femmes.

[45] SCHEERLINCK 2011-2012, p. 165.

[46] Cf. VeÏSSE 2011, p. 127-130.

[47] Sur cette perception de la condition privilégiée des femmes égyptiennes par l'historiographie, et d'une infériorité de la femme grecque à cet égard, cf. VEÏSSE 2011, p. $126-127$.

[48] Anne-Emmanuelle Veïsse a par ailleurs montré
Ce qui ressort de ces textes, c'est une insistance sur la différence de statut social et d'origine géographique entre Hérakleidès et Psénobastis dans le $P$. Enteux. 79, sur l'incapacité à travailler - et donc, à travers cela, sur le dommage économique subi - et la mise en danger de sa vie pour Philista dans le P. Enteux. 82. Bien que l'interprétation d'une opposition genrée entre les protagonistes de ces textes doive être écartée au profit des questions d'ethnicité et de dommages financiers, ces deux pétitions ne sont pas pour autant dénuées d'informations sur le statut des femmes dans I'Égypte hellénistique. Ainsi, la démarche indépendante de Philista dans le $P$. Enteux. 82 rappelle que contrairement à certains préjugés encore tenaces, qui veulent que la femme grecque d'Égypte ait disposé de moins de droits que la femme égyptienne [47], les femmes grecques pouvaient en réalité effectuer certaines actions légales et judiciaires elles-mêmes sans passer par un mari ou un parent masculin, et sans avoir à justifier cette autonomie [48].

Les préjugés sur le droit des femmes dans l'Égypte ptolémaïque ne se limitent pas aux femmes grecques : lorsqu'une femme égyptienne effectue une démarche elle-même, un éventuel veuvage ou l'absence de parent masculin encore en vie est souvent envisagé par les chercheurs. L'exemple de Chéred-Ankh dans I'archive démotique de Siout (début du II ${ }^{\mathrm{e}} \mathrm{S}$. av. J.-C.), est assez représentatif de ce phénomène [49] : dans les documents du procès qui oppose Totoès à son demi-frère Difopis, à propos de I'héritage de leur père [50], Chéred-Ankh porte plainte elle-même pour défendre les droits de Totoès, son époux, et ainsi protéger les intérêts de leur fils. Afin d'expliquer l'autonomie de cette démarche, $\mathrm{H}$. Thompson supposait que Totoès était dans l'incapacité de se représenter lui-même [51] : peut-être était-il en prison ; il est en tout cas certain qu'il était toujours en vie [52]. Si dans les plaintes de I'archive de Siout, Chéred-Ankh motive son action en disant la mener au nom de son fils, encore enfant, l'absence d'intervention de Totoès n'est

que si l'intervention du kurios est requise pour certains types de documents, cette nécessité semble être «le fait de la tradition documentaire à laquelle se rattache tel ou tel type d'acte plutôt que d'une incapacité juridique consubstantielle à la condition de la femme grecque en Égypte » (VEÏSSE 2011, p. 131).

[49] Cf. Thompson 1934 ; MANNING 2003, p. 201205 ; Manning 2010, p. 207-216; Hue-ArCÉ 2017, p. $134-137$.

[50] Totoès (I'aîné) et Difopis (le cadet) sont les deux fils de Pététymis, nés de deux mères différentes.

[51] THOMPSON 1934, p. XVI-XVII.

[52] THOMPSON 1934, p. XVI. 
pourtant pas justifiée par une incapacité à agir de cet homme. Cette archive démotique est ainsi indicatrice de la possibilité pour une femme égyptienne, membre d'une famille de dignitaires [53], d'agir au nom de son fils, en lieu et place de son époux. Comme c'est le cas de Philista dans le $P$. Enteux. 82, Chéred-Ankh ne considère pas sa situation comme exceptionnelle et devant faire l'objet d'une justification spécifique.

Les textes grecs et démotiques d'Égypte indiquent qu'il était possible pour les femmes hellènes et égyptiennes d'agir en toute autonomie, au moins en matière de dépôt de plainte [54] : la présence du kurios était néanmoins nécessaire pour les Grecques devant le tribunal, comme semble l'indiquer le parallèle du P. Gurob 2 [55].

Il faut pourtant se garder de tomber dans un autre préjugé, qui voudrait que les femmes d'Égypte aient bénéficié de conditions de vie idylliques. Ces documents qui nous présentent des femmes autonomes ne doivent pas occulter le fait que l'Égypte d'époque hellénistique demeure une société patriarcale [56], où les affaires économiques étaient la plupart du temps sous la responsabilité des hommes, même si elles pouvaient être gérées par des femmes. L'Égypte ptolémaïque n'est par ailleurs pas exempte de préjugés négatifs sur les femmes [57] : la société égyptienne n'était pas un havre de paix égalitaire. L'autonomie des femmes, loin d'être liée à la seule question du genre, était en réalité dépendante d'autres facteurs, notamment le statut social et la position dans la maisonnée.

[53] Les protagonistes du procès relaté dans les documents de l'archive de Siout sont des dignitaires rattachés à la nécropole, détenteurs de terres du temple et de liturgies. Cf. THOMPSON 1934, p. xix.

[54] L'intervention du kurios de la femme grecque demeure requise pour certains types de documents, notamment les documents contractuels ; cf. VEÏSSE 2011, p. $130-131$.

[55] Dans ce procès de Crocodilopolis, Héracléia, également accusée d'hubris, se présente en effet devant le tribunal accompagnée de son kurios. Cf. GILBART SMYLY 1921, p. $11-18$.

[56] HuEBNER 2013, p. 49, 200.

[57] Voir notamment I'analyse des textes sapientiaux démotiques menées par DieLEMAN 1998, p. 7-46, dont ressort l'image particulièrement péjorative des femmes et de leur sexualité dans le milieu clérical.

\section{BIBLIOGRAPHIE}

Bauschatz, John, 2013, Law and enforcement in Ptolemaic Egypt, Cambridge.

Boehringer, Sandra \& Sebillotte Cuchet, Violaine, 2011, Hommes et femmes dans l'antiquité grecque et romaine. Le Genre : méthodes et documents, Paris.

Boehringer, Sandra \& Sebillotte Cuchet, Violaine, 2015 « Corps, sexualité et genre dans les mondes grec et romain », Dialogues d'histoire ancienne 40, p. 83-108.

Cadell, Hélène, Clarysse, Willy \& Robic, Kennokka, 2011, Papyrus de la Sorbonne (P. Sorb. III). Nos 70-140, Paris (Papyrologica Parisina 1 ).

Clarysse, Willy \& Lanciers, Eddy, 1989, « Currency and the dating of Demotic and Greek papyri from the Ptolemaic period $\gg$, Ancient Society 20, p. 117-232.

Dieleman, Jacco, 1998, « Fear of women? Representations of women in Demotic wisdom texts », Studien zur Altägyptischen Kultur 25, p. 7-46.

FAUCHER, Thomas \& REDON, Bérangère, 2014, « Le prix de l'entrée au bain en Égypte hellénistique et romaine d'après les données textuelles et numismatiques », dans Marie-Françoise Boussac, Sylvie Denoix, Thibaud Fournet \& Bérangère Redon (éd.), 25 siècles de bain collectif en Orient. Proche-Orient, Égypte et péninsule Arabique. balaneia, thermae, hammāmāt, Actes du 3e colloque international Balnéorient, organisé par l'Institut français du Proche-Orient et la Direction Générale des Antiquités et des Musées de Syrie, Damas-Syrie/2-6 nov. 2009, Le Caire (Études Urbaines 9.3).

GilbaRT SmyLY, Josiah, 1921, Greek papyri from Gurob, Dublin (Cunningham Memoirs 12).

GuÉRAUD, Octave, 1931-1932, ENTEYEEI : requêtes et plaintes adressées au roi d'Égypte au III siècle avant J.-C., Le Caire (Publications de la Société Royale Égyptienne de Papyrologie. Textes et documents 1). 
Helmis, Andréas, 1986, Crime et châtiment dans l'Égypte ptolémäqque : recherches sur l'autonomie d'un modèle pénal, Thèse d'État, Université Paris Ouest Nanterre La Défense, Nanterre.

Hue-Arcé, Christine, 2015, La violence interpersonnelle dans la documentation égyptienne au Nouvel Empire et aux époques grecque et romaine ( $X V I^{e}-X^{e} s$. av. $n$. ère / IV $S$. $a v$. - IV $s$. de $n$. ère), Thèse de doctorat, Université de Strasbourg. Hue-ARCÉ, Christine, 2017, « Violence against women in Graeco-Roman Egypt: the contribution of Demotic documents », dans Uroš Matić \& Bo Jensen (éd.), Archaeologies of gender and violence, Oxford, p. 133-149.

Huebner, Sabine, 2013, The family in Roman Egypt. A comparative approach to intergenerational solidarity and conflict, Cambridge.

LEGRAS, Bernard, 1999, « Le corps violenté des femmes dans l'Égypte ptolémaïque d'après la documentation papyrologique », Cahiers du Centre Glotz 10, p. 225-234.

LeSQUIER, Jean, 1912, Papyrus grecs. Volume II. Papyrus de Magdôla, Paris.

LewIS, Naphtali, 1986, Greeks in Ptolemaic Egypt: case studies in the social history of the Hellenistic world, Oxford.

MANNING, Joseph G., 2003, Land and power in Ptolemaic Egypt: the structure of land tenure, Cambridge.

MANNING, Joseph G., 2010, The last pharaohs: Egypt under the Ptolemies, 305-30 BC, Princeton.

MATIĆ, Uroš, 2016, «(De)queering Hatshepsut: binary bind in archaeology of Egypt and Kingship beyond the corporeal », Journal of Archaeological Method and Theory 23, [en ligne] DOI 10.1007/s10816-016-9288-9[page consultée le 23-07-2017], p. 1-22.

Mélèze-ModrzejewskI, Joseph, 2011, « La justice des Lagides », dans Joseph Mélèze-Modrzejewski (éd.), Droit et justice dans le monde grec et hellénistique, Warsaw (The Journal of Juristic Papyrology, Supplement 10), p. 111-139.

PARCA, Marylin, 2002, «Violence by and against women in documentary papyri from Ptolemaic and Roman Egypt », dans Leon Mooren \& Henri Melaerts (éd.), Le rôle et le statut de la femme en Égypte hellénistique, romaine et byzantine ${ }_{L}$ Actes du colloque international, Bruxelles - Leuven, 27-29 novembre 1997, Leuven (Studia Hellenistica 37), p. 283-296.

Peremans, Willy, 1970, «Ethnies et classes dans l'Égypte ptolémaïque », dans Claude Nicolet (organisateur), Recherches sur les structures sociales dans l'Antiquité Classique, Caen, 25-26 avril 1969, Paris (Colloques nationaux du Centre national de la recherche scientifique), p. 213-223.

REDON, Bérangère, 2015, «Rencontres, violence et sociabilité aux bains. La clientèle des édifices balnéaires ptolémaïques », Topoi. Orient-Occident 20, p. 59-87.

RoWlANDSON, Jane, 1998, Women and society in Greek and Roman Egypt: a sourcebook, Cambridge.

SCheERLINCK, Eline, 2011-2012, «Inheritance disputes and violence in women's petitions from Ptolemaic Egypt », Papyrologica Lupiensia 20-21, p. 163-176.

TaUbensChlaG, Raphael, 1955, The Law of Graeco-Roman Egypt in the light of the papyri, 332 B.C.-A.D. 640, $2^{\mathrm{e}}$ éd. (1 $1^{\text {re }}$ éd. 1944), New York.

Tномpson, Dorothy J., 2007, « The Exceptionality of the Early Ptolemaic Fayyum », dans Mario Capasso \& Paolo Davoli (éd.), New archaeological and papyrological researches on the Fayyum: proceedings of the International Meeting of Egyptology and Papyrology. Lecce, June 8th-10th 2005, Galatina (Papyrologica Lupiensia 14), p. 303-310.

Thompson, Herbert, 1934, A family archive from Siut, from papyri in the British Museum: including an account of a trial before the Laocritae in the year B.C. 170, Oxford.

Uebel, Fritz, 1968, Die Kleruchen Ägyptens unter den ersten sechs Ptolemäern, Berlin (Abhandlungen der Deutschen Akademie der Wissenschaft zu Berlin. Klasse für Sprachen, Literatur und Kunst 3).

Ver̈sse, Anne-Emmanuelle, 2011, « Grecques et Égyptiennes en Égypte au temps des Ptolémées », Clio. Femmes, Genre, Histoire 33, p. 125-137.

Veïsse, Anne-Emmanuelle, 2012, «L'usage des ethniques dans l'Égypte du III ${ }^{\mathrm{e}}$ siècle », dans Julien Zurbach \& Laurent Capdetrey (éd.), Mobilités grecques : mouvements, réseaux, contacts en Méditerranée de l'époque archaïque à l'époque hellénistique, Bordeaux, p. 43-52.

VeÏsse, Anne-Emmanuelle, 2013, «L'expression de l'identité dans les pétitions d'époque ptolémaïque. Étude préliminaire », dans Silvia Bussi (éd.), Egitto dai Faraoni agli Arabi : atti del convegno Egitto: amministrazione, economia, società, cultura dai Faraoni agli Arabi. Milano, Università degli Studi, 7-9 gennaio 2013, Pisa - Roma, p. 81-89. 